\title{
Ultra-Cold Bosons in Optical Lattice: Time-of-Flight Imaging of Atom-Atom Correlations
}

\author{
T.A. Zaleski And T.K. KopeĆ \\ Institute of Low Temperature and Structure Research, Polish Academy of Sciences \\ POB 1410, 50-950 Wrocław 2, Poland
}

\begin{abstract}
In the present paper, we investigate a system of strongly interacting bosons confined in two-dimensional optical lattice. We propose a combination of the Bogoliubov method with the quantum rotor approach and determine the spatial atom-atom correlations. This allows us to calculate time-of-flight absorption images, which exhibit all the characteristic features present in experimental results, namely transition from superfluid peaks to Mott insulating blob.
\end{abstract}

PACS: 03.75.Kk, 05.30.Jp, 67.85.Hj

\section{Introduction}

Placing cold atoms in optical lattices allows to strongly enhance interactions between them. Because, such systems can be described by Hamiltonians that are also used for strongly correlated condensed matter manybody problems, enormous interest to study the properties of atoms in optical lattices developed recently [1]. Their advantage is obvious: they provide a clean system without impurities or defects, where almost all important parameters like lattice geometry and interaction strength can be easily tuned to almost any arbitrary value [2]. As a result, they became an almost perfect tool to test theoretical models and approximations used to solve them. As the strong correlations play a leading role in those systems the analytical studies of correlation functions are called for [3]. The atom-atom correlation function is defined by:

$$
C\left(\boldsymbol{r}, \boldsymbol{r}^{\prime}\right)=\left\langle\hat{a}(\boldsymbol{r}) \hat{a}^{\dagger}\left(\boldsymbol{r}^{\prime}\right)\right\rangle,
$$

where $\hat{a}(\boldsymbol{r})$ is a bosonic operator and $\langle\ldots\rangle$ is statistical averaging. Experimentally, the atom-atom correlation function is measured by time-of-flight absorption images $[4,5]$. The cloud of ultra-cold atoms is first suddenly released from the trapping potential. After a time of flight $t$, the position of the atoms is proportional to the momentum of the atoms in the initial cloud. Finally, an absorption image of the expanding cloud of atoms is taken by a probing laser. The resulting image provides directly the distribution of the momentum space $n(\boldsymbol{k})$. It is our goal of the presented paper, to calculate this quantity using the quantum rotor approach developed in our previous works $[6,7]$. However, in order to maintain the valid form of the correlation function, the method must be supplemented by the implementation of the Bogoliubov theory [8]. The remainder of the paper is as follows: in Section 2, we introduce the microscopic Bose-Hubbard model, which we use for the description of strongly interacting bosons in an optical lattice. In the following Section, we present our method that combines Bogoliubov approximation with quantum rotors approach. Furthermore, in Section 4, we calculate atom-atom correlation functions and resulting absorption patterns of time-offlight experiments. Finally, we conclude in the Section 5.

\section{Model Hamiltonian}

Bosons in optical lattice are described by a microscopic Bose-Hubbard Hamiltonian. The atoms are mobile in the lattice within a tight-binding scheme, while the correlation is introduced through an on-site repulsive term. The second quantized Hamiltonian is written in the form $[9,10]$ :

$$
\begin{aligned}
\hat{\mathcal{H}} & =-t \sum_{\left\langle\boldsymbol{r}, \boldsymbol{r}^{\prime}\right\rangle}\left(\hat{a}^{\dagger}(\boldsymbol{r}) \hat{a}\left(\boldsymbol{r}^{\prime}\right)+\hat{a}^{\dagger}\left(\boldsymbol{r}^{\prime}\right) \hat{a}(\boldsymbol{r})\right) \\
& +\frac{U}{2} \sum_{\boldsymbol{r}} \hat{n}^{2}(\boldsymbol{r})-\bar{\mu} \sum_{\boldsymbol{r}} \hat{n}(\boldsymbol{r}),
\end{aligned}
$$

where $t$ is a nearest neighbors tunneling matrix element responsible for hopping of bosons between neighboring sites of a regular two-dimensional (2D) lattice and $U$ is the on-site repulsive interaction of bosons. The number of bosons is controlled by a chemical potential $\mu$, while $\bar{\mu}=\mu+\frac{U}{2}$. The operators $\hat{a}^{\dagger}(\boldsymbol{r})$ and $\hat{a}\left(\boldsymbol{r}^{\prime}\right)$ create and annihilate bosons on sites $\boldsymbol{r}$ and $\boldsymbol{r}^{\prime}$, while $\left\langle\boldsymbol{r}, \boldsymbol{r}^{\prime}\right\rangle$ denotes the summation over nearest neighbors only. A total number of sites is equal to $N$ and the boson number operator $\hat{n}(\boldsymbol{r})=\hat{a}^{\dagger}(\boldsymbol{r}) \hat{a}(\boldsymbol{r})$. We concentrate on a homogeneous (translationally invariant) case, omitting the effect of the external magnetic potential that is usually superimposed on top of the optical lattice potential in order to additionally trap the atoms. The Hamiltonian 
and its descendants have been widely studied within the last years. The phase diagram and ground-state properties include the mean-field ansatz [11], strong coupling expansions [12-14], the quantum rotor approach [15], methods using the density matrix renormalization group DMRG [16-19], and quantum Monte Carlo QMC simulations [20-23].

\section{Method}

We start with the statistical sum of the system defined by Eq. (2) in a path integral form with use of complex fields $a(\boldsymbol{r} \tau)$ depending on the "imaginary time" $0 \leq \tau \leq \beta \equiv 1 / k_{B} T$, (with $T$ being the temperature) that satisfy the periodic condition $a(\boldsymbol{r} \tau)=a(\boldsymbol{r} \tau+\beta)$ :

$$
Z=\int[\mathcal{D} \bar{a} \mathcal{D} a] \mathrm{e}^{-\mathcal{S}[\bar{a}, a]},
$$

where the action $\mathcal{S}$ is equal to:

$$
\mathcal{S}[\bar{a}, a]=\int_{0}^{\beta} \mathrm{d} \tau\left(\mathcal{H}(\tau)+\sum_{\boldsymbol{r}} \bar{a}(\boldsymbol{r} \tau) \frac{\partial}{\partial \tau} a(\boldsymbol{r} \tau)\right) .
$$

The Bogoliubov approximation is based on the reduction of many-body quartic Hamiltonian to a quadratic one, which can be then diagonalized exactly. The bosonic operator is split into a Bose condensate macroscopic occupation $N_{0}=b_{0}^{2}$ and non-condensed fluctuation part $b_{d}(\boldsymbol{r})[8]:$

$$
b(\boldsymbol{r} \tau)=b_{0}+b_{d}(\boldsymbol{r} \tau) .
$$

Substituting the Eq. (5) into the action and neglecting the terms of the order higher than two in $b_{d}(\boldsymbol{r})$ operators, we obtain:

$$
\mathcal{S}=\mathcal{S}_{0}+\mathcal{S}_{1}+\mathcal{S}_{2},
$$

where $\mathcal{S}_{0}, \mathcal{S}_{1}$ and $\mathcal{S}_{2}$ are the zero, first and the second order terms containing interactions within and between both sub-systems $\left(b_{0}\right.$ and $\left.b_{d}(\boldsymbol{r} \tau)\right)$. The value of $b_{0}$ amplitude can be calculated in the saddle point:

$$
\frac{\partial \mathcal{S}\left[b_{0}\right]}{\partial b_{0}}=-t z-\bar{\mu}+U\left|b_{0}\right|^{2}=0,
$$

which results in:

$$
\left|b_{0}\right|^{2}=z \frac{t}{U}+\frac{\bar{\mu}}{U}
$$

This implies that the linear term $\mathcal{S}_{1}=0$ and the bosonic action is given by terms that are constant and quadratic in $b_{d}(\boldsymbol{r} \tau)$ :

$$
\mathcal{S}_{b}[\bar{b}, b]=\mathcal{S}_{0}+\mathcal{S}_{2} \text {. }
$$

However, this approach is valid only in the weak-coupling limit. In order to extend the description to the strongcoupling limit that is governed by phase fluctuations, we introduce the quantum rotor approach, which has already been employed for the calculation of the phase diagram of the cold bosons in optical lattice [6]. The fourth-order term in the Hamiltonian in Eq. (2) can be also decoupled using the Hubbard-Stratonovich (for details, see Refs. [6, $24]$ ), which leads to introduction of new bosonic variables that result from a local gauge transformation of original ones:

$$
a(\boldsymbol{r} \tau)=b(\boldsymbol{r} \tau) \mathrm{e}^{\mathrm{i} \phi \boldsymbol{r} \tau} .
$$

This transforms the strongly correlated bosonic system into a weakly interacting bosons, submerged into the bath of strongly fluctuating gauge potentials (which interactions are governed by the high energy scale of $U$ ). Due to transformation of variables the order parameter is defined by:

$$
\Psi_{B}=\langle a(\boldsymbol{r} \tau)\rangle=\langle b(\boldsymbol{r} \tau)\rangle \psi_{B} .
$$

Superfluidity is obtained once both the amplitude $\langle b(\boldsymbol{r} \tau)\rangle$ is non-zero and the phase (rotor) variables become stiff and coherent. This reflects the fact that all the atoms in the condensate have the same phase and form a coherent matter wave. Thus the condensate possesses a well defined phase associated with the concept of so-called spontaneously broken U(1) gauge symmetry. The phase order parameter is defined by:

$$
\psi_{B}=\left\langle\mathrm{e}^{\mathrm{i} \phi \boldsymbol{r} \tau}\right\rangle_{\phi},
$$

where $\langle\ldots\rangle_{\phi}$ is averaging over phase action to be calculated later in this section.

After the variable transformations the statistical sum becomes:

$$
Z=\int[\mathcal{D} \bar{b} \mathcal{D} b][\mathcal{D} \phi] \mathrm{e}^{-\mathcal{S}[\bar{b}, b, \phi]},
$$

with the action:

$$
\begin{aligned}
\mathcal{S} & {[\bar{b}, b, \phi]=\sum_{\boldsymbol{r}} \int_{0}^{\beta} \mathrm{d} \tau \bar{b}(\boldsymbol{r} \tau) \frac{\partial}{\partial \tau} b(\boldsymbol{r} \tau) } \\
& -t \sum_{\left\langle\boldsymbol{r}, \boldsymbol{r}^{\prime}\right\rangle} \int_{0}^{\beta} \mathrm{d} \tau\left(\mathrm{e}^{-\mathrm{i}\left(\phi\left(\boldsymbol{r}_{\tau}\right)-\phi\left(\boldsymbol{r}^{\prime} \tau\right)\right)} \bar{b}(\boldsymbol{r} \tau) b\left(\boldsymbol{r}^{\prime} \tau\right)+h . c .\right) \\
& +\sum_{\boldsymbol{r}} \int_{0}^{\beta} \mathrm{d} \tau\left(U\langle\bar{b}(\boldsymbol{r} \tau) b(\boldsymbol{r} \tau)\rangle_{b}-\bar{\mu}\right) \bar{b}(\boldsymbol{r} \tau) b(\boldsymbol{r} \tau) \\
& +\sum_{\boldsymbol{r}} \int_{0}^{\beta} \mathrm{d} \tau\left(\frac{1}{2 U} \dot{\phi}^{2}(\boldsymbol{r} \tau)+\mathrm{i} \frac{\bar{\mu}}{U} \dot{\phi}(\boldsymbol{r} \tau)\right),
\end{aligned}
$$

which are used as a departure point for obtaining phaseonly action:

$$
\mathcal{S}_{\phi}[\phi]=-\ln \int[\mathcal{D} \bar{b} \mathcal{D} b] \mathrm{e}^{-\mathcal{S}[\bar{b}, b, \phi]},
$$

with:

$$
Z=\int[\mathcal{D} \phi] \mathrm{e}^{-\mathcal{S}_{\phi}[\phi]} .
$$

In order to calculate the phase-only action, we use the following approximation:

$$
a(\boldsymbol{r} \tau)=b(\boldsymbol{r} \tau) \mathrm{e}^{\mathrm{i} \phi(\boldsymbol{r} \tau)} \approx b_{0} \mathrm{e}^{\mathrm{i} \phi(\boldsymbol{r} \tau)},
$$

where $b_{0}$ is static bosonic amplitude, which was calculated in the Bogoliubov approximation. The phase-only action can be written explicitly:

$$
\begin{gathered}
\mathcal{S}_{\phi}[\phi]=\int_{0}^{\beta} \mathrm{d} \tau\left(\sum_{\boldsymbol{r}}\left(\frac{\dot{\phi}^{2}(\boldsymbol{r} \tau)}{2 U}+\mathrm{i} \frac{\bar{\mu}}{U} \dot{\phi}(\boldsymbol{r} \tau)\right)\right. \\
\left.-J \sum_{\left\langle\boldsymbol{r}, \boldsymbol{r}^{\prime}\right\rangle} \cos \left(\phi(\boldsymbol{r} \tau)-\phi\left(\boldsymbol{r}^{\prime} \tau\right)\right)\right),
\end{gathered}
$$

where $J=t\left|b_{0}\right|^{2}$ represents the stiffness for the phase field. Since, the phase action is non-linear in the phase variables $\phi(\boldsymbol{r} \tau)$ and the statistical sum in Eq. (16) can- 
not be calculated exactly, we are bound to make further approximations. First, we introduce a new uni-modular collective field $z(\boldsymbol{r} \tau)=\mathrm{e}^{\mathrm{i} \phi(\boldsymbol{r} \tau)}$. Then, in order to make to model solvable, we implement the constraint for $z(\boldsymbol{r} \tau)$ length that preserves uni-modularity only on average (see Ref. [25]):

$$
\begin{aligned}
\delta & \left(N-\sum_{\boldsymbol{r}} \bar{z}(\boldsymbol{r} \tau) z(\boldsymbol{r} \tau)\right) \\
= & \int \mathrm{d} \lambda \mathrm{e}^{N \lambda-\lambda \sum_{\boldsymbol{r}} \bar{z}\left(\boldsymbol{r}_{\tau}\right) z\left(\boldsymbol{r}_{\tau}\right) .}
\end{aligned}
$$

This introduces a Lagrange multiplier $\lambda$. Substituting the constraint into Eq. (16) and integrating by the cumulant expansion over the phase variables, the partition function reads:

$$
Z=\int[\mathcal{D} \bar{z} \mathcal{D} z] \mathrm{d} \lambda \mathrm{e}^{N \lambda-\mathcal{S}_{z}[\bar{z}, z]},
$$

In the thermodynamic limit $(N \rightarrow \infty)$, the integral (20) can be performed exactly by the saddle-point method. The quantum rotor action:

$$
\begin{aligned}
& \mathcal{S}_{z}[\bar{z}, z]=\sum_{\left\langle\boldsymbol{r}, \boldsymbol{r}^{\prime}\right\rangle} \int_{0}^{\beta} \mathrm{d} \tau \mathrm{d} \tau^{\prime}\left(\left(\lambda \delta_{\boldsymbol{r} \boldsymbol{r}^{\prime}}-t b_{0}^{2}\right) \delta\left(\tau-\tau^{\prime}\right)\right. \\
& \left.\quad+\delta_{\boldsymbol{r} \boldsymbol{r}^{\prime}} K^{-1}\left(\tau-\tau^{\prime}\right)\right) \bar{z}(\boldsymbol{r} \tau) z\left(\boldsymbol{r}^{\prime} \tau^{\prime}\right),
\end{aligned}
$$

where $K^{-1}\left(\tau-\tau^{\prime}\right)$ is a phase correlator, which includes dynamic effect of the $\mathrm{U}(1)$ phase field:

$$
K^{-1}\left(\tau-\tau^{\prime}\right)=\left\langle\mathrm{e}^{\mathrm{i} \phi(\boldsymbol{r} \tau)-\mathrm{i} \phi\left(\boldsymbol{r} \tau^{\prime}\right)}\right\rangle_{0},
$$

with the average

$$
\langle\ldots\rangle_{0}=\frac{\int[\mathcal{D} \phi] \ldots \mathrm{e}^{-\mathcal{S}_{0}[\phi]}}{\int[\mathcal{D} \phi] \mathrm{e}^{-\mathcal{S}_{0}[\phi]}},
$$

that is taken only over non-interacting quantum rotors:

$$
\mathcal{S}_{0}=\sum_{\boldsymbol{r}} \int_{0}^{\beta} \mathrm{d} \tau\left(\frac{\dot{\phi}^{2}(\boldsymbol{r} \tau)}{2 U}+\mathrm{i} \frac{\bar{\mu}}{U} \dot{\phi}(\boldsymbol{r} \tau)\right) .
$$

The Fourier transform of the correlator in Eq. (22) in the zero-temperature limit reads:

$$
K^{-1}\left(\omega_{l}\right)=\frac{U}{4}-U\left(v\left(\frac{\bar{\mu}}{U}\right)+\frac{\mathrm{i} \omega_{l}}{U}\right)^{2},
$$

where the Bose-Matsubara frequencies are $\omega_{\ell}=2 \pi \ell / \beta$ and $\ell=0, \pm 1, \pm 2, \ldots$ Furthermore, $v(x)=x-[x]-\frac{1}{2}$, and $[x]$ is the floor function, which gives the greatest integer less then or equal to $x$. The function $v(x)$ allows to calculate the zero-temperature value of the correlator without demanding summation over winding numbers. The phase order parameter in the quantum rotor model can be written as:

$$
1-\psi_{B}^{2}=\frac{1}{N} \sum_{\boldsymbol{r}} \int_{0}^{\beta} \mathrm{d} \tau\langle\bar{z}(\boldsymbol{r} \tau) z(\boldsymbol{r} \tau)\rangle,
$$

which fixes value of the phase order parameter $\psi_{B}$. The Lagrange multiplier saddle-point value "sticks" at criticality to the value $\lambda_{0}$ given by

$$
\lambda_{0}-J(\boldsymbol{k}=0)+K^{-1}\left(\omega_{\ell=0}\right)=0,
$$

and obeys the Eq. (27) in the whole low temperature ordered phase.

\section{Correlation function and time-of-flight patterns}

The correlation function in Eq. (1) can be written as a product of two correlation functions of amplitude and rotor fields:

$$
C(\boldsymbol{R}) \equiv C_{z}(\boldsymbol{R}) C_{b}(\boldsymbol{R}),
$$

where

$$
\begin{aligned}
& C_{z}(\boldsymbol{R}) \equiv C_{z}\left(\boldsymbol{r} \tau ; \boldsymbol{r}^{\prime} \tau\right)=\left\langle z(\boldsymbol{r} \tau) \bar{z}\left(\boldsymbol{r} \tau^{\prime}\right)\right\rangle_{z}, \\
& C_{b}(\boldsymbol{R}) \equiv C_{b}\left(\boldsymbol{r} \tau ; \boldsymbol{r}^{\prime} \tau\right)=\left\langle b(\boldsymbol{r}) \bar{b}\left(\boldsymbol{r}^{\prime}\right)\right\rangle_{b},
\end{aligned}
$$

and $\boldsymbol{R}=\boldsymbol{r}-\boldsymbol{r}^{\prime}$ (since, the system is homogeneous, the correlation function depends only on distance, not the specific position of $\boldsymbol{r}$ and $\boldsymbol{r}^{\prime}$ ). The averagings appearing in Eq. (29) are defined by:

$$
\begin{aligned}
\langle\ldots\rangle_{z} & =\frac{\int[\mathcal{D} \bar{z} \mathcal{D} z] \ldots \mathrm{e}^{-\mathcal{S}_{z}[\bar{z}, z]}}{\int[\mathcal{D} \bar{z} \mathcal{D} z] \mathrm{e}^{-\mathcal{S}_{z}[\bar{z}, z]}}, \\
\langle\ldots\rangle_{b} & =\frac{\int[\mathcal{D} \bar{b} \mathcal{D} b] \ldots \mathrm{e}^{-\mathcal{S}_{b}[\bar{b}, b]}}{\int[\mathcal{D} \bar{b} \mathcal{D} b] \mathrm{e}^{-\mathcal{S}_{b}[\bar{b}, b]}},
\end{aligned}
$$

where $\mathcal{S}_{z}[\bar{z}, z]$ and $\mathcal{S}_{b}[\bar{b}, b]$ are given in Eqs. (21) and (9), respectively. As a result, the correlation function in the real space splits into a product of averages from bosonic and phase sectors. The Green's function in the bosonic sector results from the action in Eq. (9) reads:

$$
G_{b}\left(\boldsymbol{k} \omega_{m}\right)=b_{0}^{2}+G_{b d}^{22}\left(\boldsymbol{k} \omega_{m}\right),
$$

where:

$$
\begin{aligned}
& G_{b d}\left(\boldsymbol{k} \omega_{m}\right) \\
& =\left[\begin{array}{cc}
\frac{2 t\left(\varepsilon_{\mathbf{0}}-\varepsilon_{\boldsymbol{k}}\right)+U\left|b_{0}\right|^{2}-\mathrm{i} \omega_{l}}{E_{\boldsymbol{k}}^{2}-\left(\mathrm{i} \omega_{l}\right)^{2}} & -\frac{U\left|b_{0}\right|^{2}}{E_{\boldsymbol{k}}^{2}-\left(\mathrm{i} \omega_{l}\right)^{2}} \\
-\frac{U\left|b_{0}\right|^{2}}{E_{\boldsymbol{k}}^{2}-\left(\mathrm{i} \omega_{l}\right)^{2}} & \frac{2 t\left(\varepsilon_{\mathbf{0}}-\varepsilon_{\boldsymbol{k}}\right)+U\left|b_{0}\right|^{2}+\mathrm{i} \omega_{l}}{E_{\boldsymbol{k}}^{2}-\left(\mathrm{i} \omega_{l}\right)^{2}}
\end{array}\right],
\end{aligned}
$$

and

$$
E_{\boldsymbol{k}}=\sqrt{2 t\left(\frac{z}{2}-\varepsilon_{\boldsymbol{k}}\right)\left(2 t\left(\frac{z}{2}-\varepsilon_{\boldsymbol{k}}\right)+2 U\left|b_{0}\right|^{2}\right)}
$$

with a dispersion for a simple square lattice:

$$
\varepsilon_{\boldsymbol{k}}=\cos \left(a k_{x}\right)+\cos \left(a k_{y}\right) \text {. }
$$

On the other hand, the phase sector leads to the phase Green's function resulting from rotor action in Eq. (21):

$$
\begin{gathered}
G_{\phi}\left(\boldsymbol{k} \omega_{m}\right)=\left\langle z\left(\boldsymbol{k} \omega_{m}\right) \bar{z}\left(\boldsymbol{k} \omega_{m}\right)\right\rangle \\
=\frac{1}{\lambda_{0}-2 t b_{0}^{2} \varepsilon_{\boldsymbol{k}}+K^{-1}\left(\omega_{m}\right)} .
\end{gathered}
$$

As a result, the correlation function finally reads:

$$
C(\boldsymbol{R})=G_{\phi}(\boldsymbol{R})\left(\left|b_{0}\right|^{2}+G_{b d}(\boldsymbol{R})\right) .
$$

The phase coherence properties of the cold atoms in optical lattice resulting from their correlations can be observed in time of flight absorption images. In the superfluid state the atoms are coherent, sharing the same phase. As a result, after being released from the trap and the optical lattice they preserve the coherence, which manifests as coherence peaks on the time of flight images that reflect the symmetry of the optical lattice. On the 
other hand, lack of long range order of the Mott insulating state results in broad blob-like maximum. To identify the ordered state, we determine the the momentum distribution of particle number $n(\boldsymbol{k})$, a quantity of basic interest that encodes the strong correlations of the system:

$$
n(\boldsymbol{k})=\sum_{\boldsymbol{R}} C(\boldsymbol{R}) \mathrm{e}^{\mathrm{i} \boldsymbol{k} \boldsymbol{R}} .
$$

Since, in time of flight experiments the expansion of the atoms after being released from the trap is mostly ballistic, the momentum dependence of particle numbers is maintained. In our theory, using the Eqs. (28)-(35), we have:

$$
\begin{aligned}
& n(\boldsymbol{k})=\left\langle a_{\boldsymbol{k}}^{\dagger}(\tau) a_{\boldsymbol{k}}(\tau)\right\rangle \\
& \quad=m_{0} G_{b d}(\boldsymbol{k})+b_{0} G_{\phi d}(\boldsymbol{k}) \\
& \quad+\frac{1}{N} \sum_{\boldsymbol{k}^{\prime}} G_{b d}\left(\boldsymbol{k}^{\prime}\right) G_{\phi d}\left(\boldsymbol{k}-\boldsymbol{k}^{\prime}\right),
\end{aligned}
$$

where:

$$
\begin{aligned}
G_{b d}(\boldsymbol{k}) & =\frac{1}{2}\left(-1+\frac{2 t\left(\varepsilon_{0}-\varepsilon_{\boldsymbol{k}}\right)+n_{0} U}{E_{\boldsymbol{k}}} \operatorname{coth} \frac{\beta E_{\boldsymbol{k}}}{2}\right), \\
G_{\phi d}(\boldsymbol{k}) & =\frac{\operatorname{coth}\left(\frac{\beta U}{2}\left(\Omega_{\boldsymbol{k}}+v\left(\frac{\bar{\mu}}{U}\right)\right)\right)}{4 \Omega_{\boldsymbol{k}}} \\
+ & \frac{\operatorname{coth}\left(\frac{\beta U}{2}\left(\Omega_{\boldsymbol{k}}-v\left(\frac{\bar{\mu}}{U}\right)\right)\right)}{4 \Omega_{\boldsymbol{k}}}
\end{aligned}
$$

with:

$$
\Omega_{\boldsymbol{k}}=\sqrt{2 \frac{t b_{0}^{2}}{U}\left(\varepsilon_{0}-\varepsilon_{\boldsymbol{k}}\right)+v^{2}\left(\frac{\bar{\mu}}{U}\right)+\frac{\delta \lambda}{U}} .
$$

The interference pattern observed after release of the atom cloud from the optical lattice depends not only on the momentum distribution of the particles, but also on the geometry of the optical lattice. As a result, the density distribution of the expanding cloud after time $t$ can be represented as follows [26-28]:

$$
n(\boldsymbol{r})=\left.\left(\frac{m}{\hbar t}\right)^{3}|W(\boldsymbol{k})|^{2} n(\boldsymbol{k})\right|_{\boldsymbol{k}=\frac{m \boldsymbol{r}}{\hbar t}},
$$

where $m$ is the atomic mass. The effect of the lattice enters through $W(\boldsymbol{k})$, which is the Fourier transform of the Wannier function in the lowest Bloch band. Typically, the trapping potential is well approximated by a harmonic function so that the envelope has the Gaussian form:

$$
\left|W\left(\frac{m \boldsymbol{r}}{\hbar t}\right)\right|^{2} \approx \frac{1}{\pi^{3 / 2} w_{t}} \exp \left(-\frac{\boldsymbol{r}^{2}}{w_{t}^{2}}\right),
$$

where $w_{t}=\hbar t / m w_{0}$ with $w_{0}$ being the size of the on-site Wannier function. Therefore, in order to compare the interference pattern with experiments, we have to calculate $n(\boldsymbol{r})$. The results are presented in Figs. 1 and 2. In the superfluid phase, the sharp peaks emerge denoting longrange phase coherence. In the Mott phase, the momen-
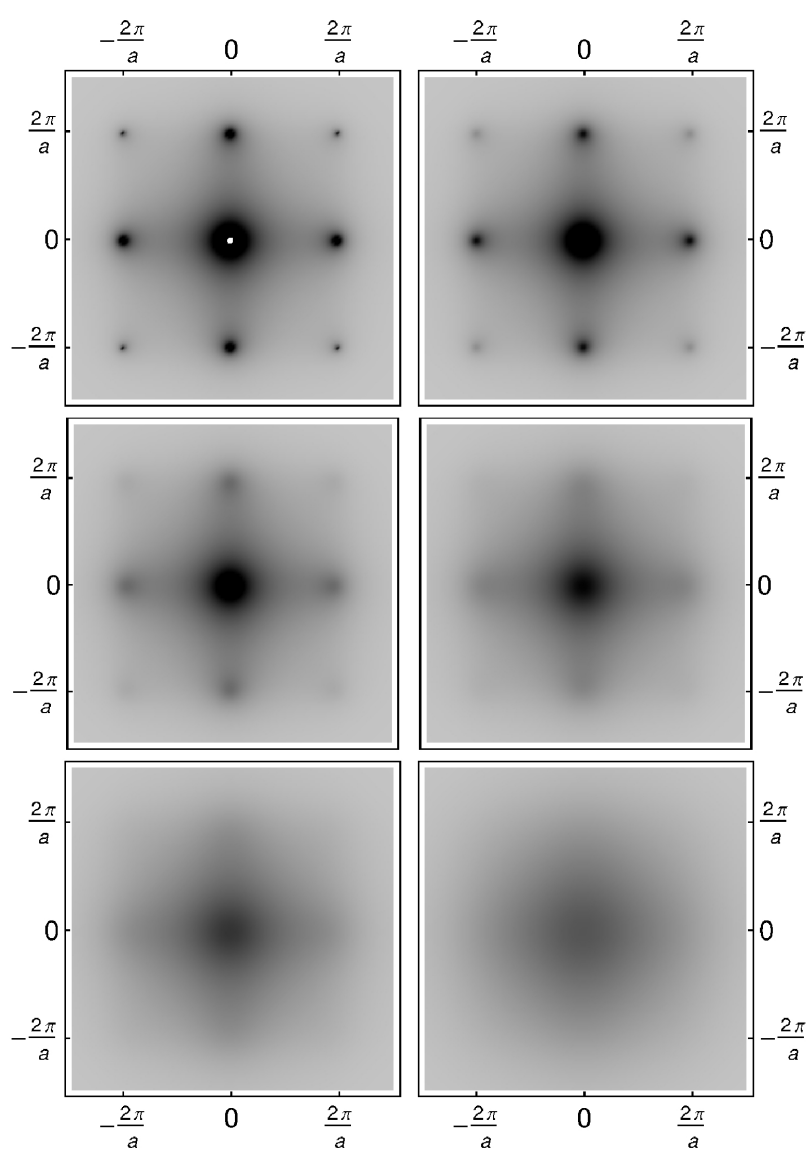

Fig. 1. Simulation of time-of-flight absorption images (from. Eq. (41)) for various interactions strength $t / U$ : transition from superfluid (top-left, $t / U=0.085$ ) to Mott phase (top-right to the bottom-right $t / U=$ $0.085,0.07,0.055,0.04,0.025,0.01$, respectively), for $\mu / U=0.5$.

tum distribution becomes a broad, featureless maximum. The data in Figs. 1 and 2 is presented in the scaled form in the units of $m \boldsymbol{r} / \hbar t$. The results are in good agreement with experimental findings.

\section{Conclusions}

In the present paper, we have studied the atom-atom correlations of cold bosons loaded in a two dimensional optical lattice. In the weak-coupling regime, the Bogoliubov theory is valid $(U / t \ll 1)$. In this approach, quasiparticle states correspond to solutions of the approximate Hamiltonian with a plane-wave character. However, as interactions become stronger, the quantum correlations become important and the exact eigenstates of the system do not necessarily have the simple plane wave character any more. As a result, in the strong interaction limit at a critical value of $U / t$ the system exhibits transition from superfuid to Mott-insulator state. Since, this transition is driven by phase fluctuations, we developed an approach that goes beyond the simple Bogoliubov approach. It incorporates the phase degrees of freedom via 
the quantum rotor approach. We have separated the problem into the amplitude of the Bose field and the fluctuating phase that was absent in the original Bogoliubov problem. Subsequently, the functional formulation of this problem was shown to be a powerful tool that incorporates properly the interaction aspects characteristic of the quantum phase dynamics. This formalism provides a useful framework, where the one particle correlation functions are treated self-consistently and permits us to test and simulate Bose-Hubbard Hamiltonian with a whole range of phenomena. Furthermore, we have calculated atom-atom correlation functions and used them to determine the absorption images of time-of-flight experiments. We found that all the characteristic features visible in the experimental results are present, namely sharp coherence peaks in the superfluid state that change to a broad maximum in the Mott insulating state.

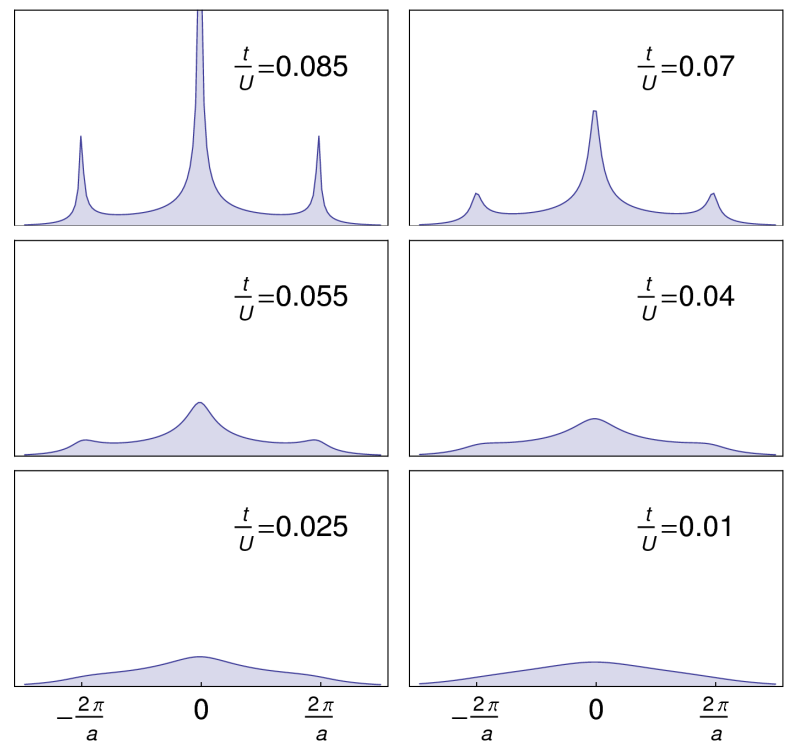

Fig. 2. Cross-section of the simulated time-of-flight absorption images (from. Eq. (41)) for various interactions strength $t / U$ : transition from superfluid (top-left, $t / U=0.085$ ) to Mott phase (top-right to the bottomright $t / U=0.085,0.07,0.055,0.04,0.025,0.01$, respectively), for $\mu / U=0.5$.

\section{Acknowledgments}

We would like to acknowledge support from Polish Ministry of Science and Higher Education (Grant No. N N202 045537).

\section{References}

[1] M. Greiner, O. Mandel, T. Esslinger, T.W. Hänsch, I. Bloch, Nature 415, 39 (2002).

[2] I. Bloch, Nat. Phys. 1, 23 (2005).

[3] I. Hen, M. Rigol, Phys. Rev. A 82, 043634 (2010).

[4] F. Gerbier, A. Widera, S. Fölling, O. Mandel, T. Gericke, I. Bloch, Phys. Rev. A 72, 053606 (2005).

[5] A. Hoffmann, A. Pelster, Phys. Rev. A 79, 053623 (2009).

[6] T.P. Polak, T.K. Kopeć, Phys. Rev. B 76, 094503 (2007).

[7] T.A. Zaleski, T.P. Polak, Phys. Rev. A 83, 023607 (2011).

[8] N.N. Bogoliubov, J. Phys. (USSR) 11, 23 (1947).

[9] M.P.A. Fisher, P.B. Weichman, G. Grinstein, D.S. Fisher, Phys. Rev. B 40, 546 (1989).

[10] S. Sachdev, Quantum Phase Transitions, Cambridge University Press, Cambridge 1999.

[11] J.K. Freericks, H. Monien, Phys. Rev. B 53, 2691 (1996).

[12] N. Elstner, H. Monien, Phys. Rev. B 59, 12184 (1999).

[13] J.K. Freericks, H.R. Krishnamurthy, Y. Kato, N. Kawashima, N. Trivedi, Phys. Rev. A 79, 053631 (2009).

[14] T.P. Polak, T.K. Kopeć, J. Phys. B 42, 095302 (2009).

[15] T.D. Kühner, H. Monien, Phys. Rev. B 58, R14741 (1998).

[16] S. Rapsch, U. Schollwöck, W. Zwerger, Europhys. Lett. 46, 559 (1999).

[17] C. Kollath, U. Schollwöck, J. von Delft, W. Zwerger, Phys. Rev. A 69, 031601(R) (2004).

[18] C. Kollath, A.M. Läuchli, E. Altman, Phys. Rev. Lett. 98, 180601 (2007).

[19] G.G. Batrouni, R.T. Scalettar, Phys. Rev. B 46, 9051 (1992).

[20] S. Wessel, F. Alet, M. Troyer, G.G. Batrouni, Phys. Rev. A 70, 053615 (2004).

[21] B. Capogrosso-Sansone, N.V. Prokof'ev, B.V. Svistunov, Phys. Rev. B 75, 134302 (2007).

[22] B. Capogrosso-Sansone, Ş.G. Söyler, N. Prokof'ev, B. Svistunov, Phys. Rev. A 77, 015602 (2008).

[23] T.K. Kopeć, Phys. Rev. B 70, 054518 (2004).

[24] T.K. Kopeć, J.V. José, Phys. Rev. B 60, 7473 (1999).

[25] W. Zwerger, J. Opt. B: Quantum Semiclassical Opt. 5, S9 (2003).

[26] V.A. Kashurnikov, N.V. Prokof'ev, B.V. Svistunov, Phys. Rev. A 66, 031601R (2002).

[27] P. Pedri, L. Pitaevskii, S. Stringari, C. Fort, S. Burger, F.S. Cataliotti, P. Maddaloni, F. Minardi, M. Inguscio, Phys. Rev. Lett. 87, 220401 (2001).

[28] K. Günter, T. Stöferle, H. Moritz, M. Köhl, T. Esslinger, Phys. Rev. Lett. 96, 180402 (2006). 